\title{
New constraints on the magnetic field in cosmic web filaments ${ }^{\star}$
}

\author{
N. Locatelli ${ }^{1,2,3}$, F. Vazza ${ }^{1,2,4}$, A. Bonafede ${ }^{1,2,4}$, S. Banfi ${ }^{1,2}$, G. Bernardi ${ }^{2,5,6}$, C. Gheller ${ }^{2}$, \\ A. Botteon ${ }^{7}$, and T. Shimwell ${ }^{7,8}$
}

\author{
1 Dipartimento di Fisica e Astronomia, Universitá di Bologna, Via Gobetti 93/2, 40122 Bologna, Italy \\ e-mail: nicola.locatelli2@unibo.it \\ 2 Istituto di Radioastronomia, INAF, Via Gobetti 101, 40122 Bologna, Italy \\ 3 Max-Planck-Institut für Extraterrestrische Physik (MPE), Giessenbachstrasse 1, 85748 Garching bei München, Germany \\ e-mail: nlocat@mpe.mpg.de \\ ${ }^{4}$ Hamburger Sternwarte, University of Hamburg, Gojenbergsweg 112, 21029 Hamburg, Germany \\ 5 Department of Physics and Electronics, Rhodes University, PO Box 94, Grahamstown 6140, South Africa \\ 6 South African Radio Astronomy Observatory, Black River Park, 2 Fir Street, Observatory, Cape Town 7925, South Africa \\ 7 Leiden Observatory, Leiden University, PO Box 9513, 2300 RA Leiden, The Netherlands \\ 8 ASTRON, The Netherlands Institute for Radio Astronomy, Postbus 2, 7990 AA Dwingeloo, The Netherlands
}

Received 10 February 2021 / Accepted 15 May 2021

\section{ABSTRACT}

\begin{abstract}
Strong accretion shocks are expected to illuminate the warm-hot intergalactic medium encompassed by the filaments of the cosmic web, through synchrotron radio emission. Given their high sensitivity, large low-frequency radio facilities may already be able to detect signatures of this extended radio emission from the region between two close and massive galaxy clusters. In this work we exploit the non-detection of such diffuse emission by deep observations of two pairs of relatively close $(\simeq 10 \mathrm{Mpc})$ and massive $\left(M_{500} \geq 10^{14} M_{\odot}\right)$ galaxy clusters using the LOw-Frequency ARray. By combining the results from the two putative inter-cluster filaments, we derive new independent constraints on the median strength of intergalactic magnetic fields: $B_{10 \mathrm{Mpc}}<2.5 \times 10^{2} \mathrm{nG}(95 \%$ confidence level). Based on cosmological simulations and assuming a primordial origin of the $B$-fields, these estimates can be used to limit the amplitude of primordial seed magnetic fields: $B_{0} \leq 10 \mathrm{nG}$. We recommend the observation of similar cluster pairs as a powerful tool to set tight constraints on the amplitude of extragalactic magnetic fields.
\end{abstract}

Key words. magnetic fields - acceleration of particles - galaxies: clusters: general

\section{Introduction}

On the largest scales of the Universe ( $\geq 10 \mathrm{Mpc})$, galaxy groups and clusters are connected by elongated distributions of galaxies called filaments and sheets which are also believed to be permeated by diffuse gas, and possibly by magnetic fields. To date, a straightforward and direct detection of intergalactic medium (IGM) and an intergalactic magnetic field (IGMF) has been prevented by the very low density of the plasma $\left(n_{\mathrm{IGM}} \leq 10^{-4} \mathrm{~cm}^{-3}\right)$ and its relatively low temperature $\left(T_{\mathrm{IGM}} \leq 10^{7} \mathrm{~K}\right)$. However, increasing evidence (Nicastro et al. 2018; Macquart et al. 2020; Tanimura et al. 2020) is confirming the long-lived expectations for the warm-hot gas phase of the IGM (WHIM, with $T_{\text {WHIM }} \sim 10^{5}-10^{7}, n_{\text {WHIM }} \sim 10^{-5}-10^{-4}$ ) to contain up to half of the baryon content at low redshifts (Cen \& Ostriker 1999; Davé et al. 2001; Reiprich et al. 2021).

Accretion shocks are believed to reside along and within the filaments of the cosmic web and at the outskirts of galaxy clusters. These shocks are expected to amplify the magnetic fields and to accelerate particles up to relativistic energies (Ryu et al. 2008). Their presence might then enable the detection of the WHIM through its synchrotron emission signature at radio wavelengths, and indeed the direct observation of the tip of the iceberg of this diffuse emission has already been

* The code used to produce the simulations discussed in this paper is public (https://enzo-project.org). Significant subsets of the simulations are publicly available at https://cosmo simfrazza.myfreesites.net/the_magnetic_cosmic_web, while larger data sets can be shared upon request. obtained at radio frequencies (Govoni et al. 2019; Botteon et al. 2020a). In these few cases the plasma conditions are still hotter and denser than was expected for the WHIM, and the detected emission lays within the clusters virial radii. Further works in this direction will be helped in the near future by the promising new and upcoming radio facilities (the next generation Very Large Array, ngVLA; the Karoo Array Telescope, MeerKAT; the square kilometer array SKA-mid) and especially at very low frequencies (the LOw-Frequency, ARray LOFAR; the Murchison Widefield Array, MWA; SKA-low) where the emission should be brighter up to further out the clusters virial radii thanks to the expected spectral behaviour as $S_{v} \propto v^{-1}$ with respect to frequency $v$ (Vazza et al. 2015). A way to overcome sensitivity limitations is to quantify the Faraday effect induced by magneto-ionised plasma along the line of sight to a polarised background radio source, and to build a tomography of the WHIM by means of a grid of background sources (see Akahori et al. 2014; Vacca et al. 2016). A thorough exploitation of this method currently suffers from the lack of large and dense grids of polarised sources; however, it is expected to provide important results thanks to the upcoming radio facilities (Locatelli et al. 2018). Recent upper limits on the IGMF intensity and scale were derived from the cross-correlation of diffuse radio synchrotron emission with the underlying galaxy distribution (Vernstrom et al. 2017; Brown et al. 2017) by crosscorrelating the difference in rotation measures of physically related pairs of extended radio galaxies, compared with that derived from randomly paired close lobes (Vernstrom et al. 2019; O'Sullivan et al. 2020; Stuardi et al. 2020) and by 
Table 1. Main parameters of the two pairs of galaxy clusters observed in this work, based on the Meta-Catalogue of X-ray Detected Clusters of Galaxies (MCXC; Piffaretti et al. 2011).

\begin{tabular}{lccccccccc}
\hline \hline Cluster name & $\begin{array}{c}\mathrm{RA} \\
{[\mathrm{h}, \mathrm{m}, \mathrm{s}]}\end{array}$ & $\begin{array}{c}\mathrm{Dec} \\
{\left[{ }^{\circ},{ }^{\prime \prime}\right]}\end{array}$ & $z$ & $\begin{array}{c}L_{\mathrm{X}} \\
{\left[\mathrm{erg} \mathrm{s}^{-1}\right]}\end{array}$ & $\begin{array}{c}M_{500} \\
{\left[M_{\odot}\right]}\end{array}$ & $\begin{array}{c}R_{500} \\
{[\mathrm{Mpc}]}\end{array}$ & $\frac{d_{3 \mathrm{D}}}{\left(R_{1}+R_{2}\right)}$ & $\begin{array}{c}\text { Angular separation } \\
{\left[{ }^{\circ}\right]}\end{array}$ & $\begin{array}{c}L_{\mathrm{fila}} \\
{[\mathrm{Mpc}]}\end{array}$ \\
\hline RXCJ1155.3+2324 & 115518 & +232427 & 0.142 & $6.04 \times 10^{44}$ & $5.60 \times 10^{14}$ & 1.19 & 7.0 & 0.93 & 14 \\
RXCJ1156.9+2415 & 115658 & +241529 & 0.139 & $1.50 \times 10^{44}$ & $2.40 \times 10^{14}$ & 0.90 & 7.0 & 0.93 & 14 \\
\hline RXCJ1659.7+3236 & 165944 & +323649 & 0.101 & $1.12 \times 10^{44}$ & $2.04 \times 10^{14}$ & 0.87 & 13.3 & 1.57 & 25 \\
RXCJ1702.7+3403 & 170242 & +340343 & 0.095 & $4.04 \times 10^{44}$ & $4.49 \times 10^{14}$ & 1.01 & 13.3 & 1.57 & 25 \\
\hline
\end{tabular}

Notes. In the last three columns we give the 3D distance between the two cluster centres (normalised by the radius of the two clusters), the angular separation of the two cluster centres, and the 3D length of the filament (considering the cluster-to-cluster distance).

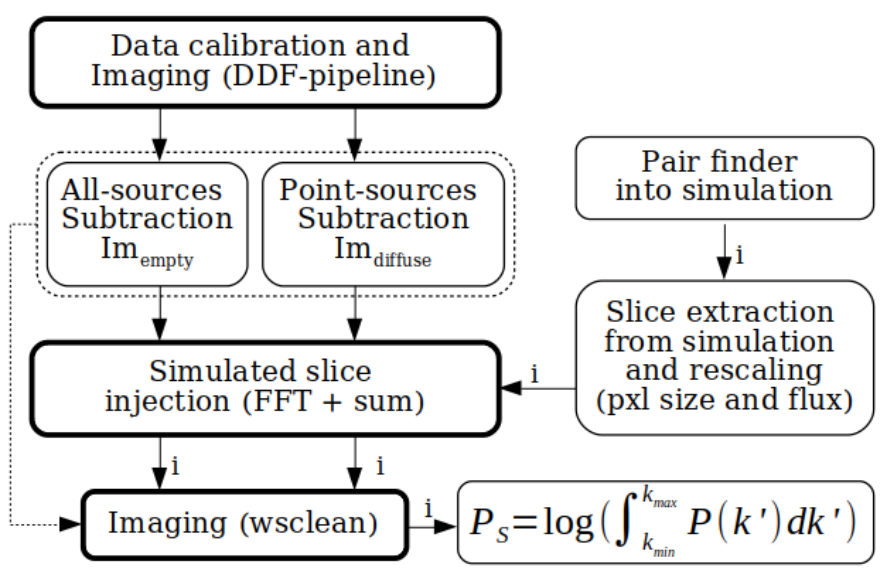

Fig. 1. Diagram of the method outlined in Sect. 2. The thick boxes highlight the most computationally expensive steps. Links labelled ' $i$ ' are computed iteratively over the simulated pairs.

stacking full sky low-frequency radio images of close red luminous galaxy pairs (Vernstrom et al. 2021).

The question arises of why it is important to assess the IGMF properties in the cosmic web at late times. The magnetic fields in galaxies and galaxy clusters, commonly observed today, arise from strong amplification from efficient magnetohydrodynamic (MHD) small-scale mechanisms (Ryu et al. 2008) that are responsible for a fast saturation of the fields, thus erasing information of their initial conditions, and in turn their origins (Beresnyak \& Miniati 2016). Instead, in the WHIM environment the amplification of primordial magnetic fields is found in simulations to be mainly driven by the field compression as its lines freeze into the plasma plus the contribution of small scale shocks. These mechanisms do not bring the field to saturation, and provide a tool of assessing the history and original conditions of the field by means of the level of magnetisation observed today (Vazza et al. 2014, 2015; Donnert et al. 2018). For the above reasons it is crucial to constrain the magnetic field in the WHIM in order to determine the original scenario for the large-scale magnetic field origin and evolution in the Universe.

Cosmological MHD simulations predict the intensity of the IGMF at low redshift to be in the range between 1 and $100 \mathrm{nG}$ (Dolag et al. 1999; Brüggen et al. 2005; Vazza et al. 2017). In this paper we introduce a novel method for a robust inference of an upper limit on the initial $B_{0}$ and current $B$ values of the IGMF within the large-scale filaments of the cosmic web. The method explores the amount of diffuse emission detected at $144 \mathrm{MHz}$ with the LOw Frequency ARray (LOFAR) telescope along the direction connecting two different pairs of galaxy clusters.
We outline the method used to explore the upper limits on the IGMF into cosmological filaments in Sect. 2; we show its results in Sect. 3 and discuss their assumptions and implications in Sect. 4; and we draw our conclusions in Sect. 5. Throughout this work we assume a $\Lambda \mathrm{CDM}$ cosmological model with baryonic, dark matter, and dark energy density parameters $\Omega_{\mathrm{BM}}=0.0455$, $\Omega_{\mathrm{DM}}=0.2265$, and $\Omega_{\Lambda}=0.728$, respectively, and a Hubble constant $H_{0}=70.2 \mathrm{~km} \mathrm{~s}^{-1} \mathrm{Mpc}^{-1}$.

\section{Method}

In order to look for large-scale emission from the cosmic web, we observed pairs of galaxy clusters and the putative inter-cluster filaments connecting them. The cluster pairs were selected from the Meta-Catalogue of X-ray Detected Clusters of Galaxies $^{1}$ (MCXC, Piffaretti et al. 2011) by applying cuts in declination ( $\delta \geq 10 \mathrm{deg})$, redshift $(z \leq 0.3)$, and maximum angular separation $(\theta \leq 5 \mathrm{deg})$. These values were tailored to the proposed LOFAR observations. The two most promising pairs that, according to cosmological simulations, maximise the probability of a physical connection between the clusters in terms of total mass and separation (real and projected), were proposed and observed at the LOFAR during Cycle 9 (Proposal Id:LC9_020). The most important properties of the two observed pairs of clusters are given in Table 1.

In a nutshell, after calibrating, imaging, and removing contaminating sources from the LOFAR data, we quantify the confidence of having observed (or not) diffuse emission from the inter-cluster filaments. We inject simulated diffuse emission produced by large-scale accretion shocks $(\geq \mathrm{Mpc})$, into the original radio visibility data $(u v w)$ for a large subset $(O(100))$ of simulated filaments and cluster pairs and image them, as done for the real observations.

In this section we provide further details of the analysis performed on the actual observations, the simulated data set prepared for the injection, and the injection procedure (see diagram in Fig. 1).

\subsection{LOFAR radio observations}

We observed the two cluster pairs RXCJ1659.7+3236-RXCJ $1702.7+3403$ (hereafter RXC_J1659-J1702) and RXCJ11 55.3+2324-RXCJ1156.9+2415 (hereafter RXC_J1155-J1156) using LOFAR. The fields containing these two targets were co-observed with two pointings of the LOFAR Two-metre Sky Survey (LoTSS; Shimwell et al. 2017) taking advantage of the multi-beam capabilities of LOFAR. The observing set-up of our observations thus follows that of LoTSS,

1 http://heasarc.gsfc .nasa.gov/W3Browse/all/mcxc.html 


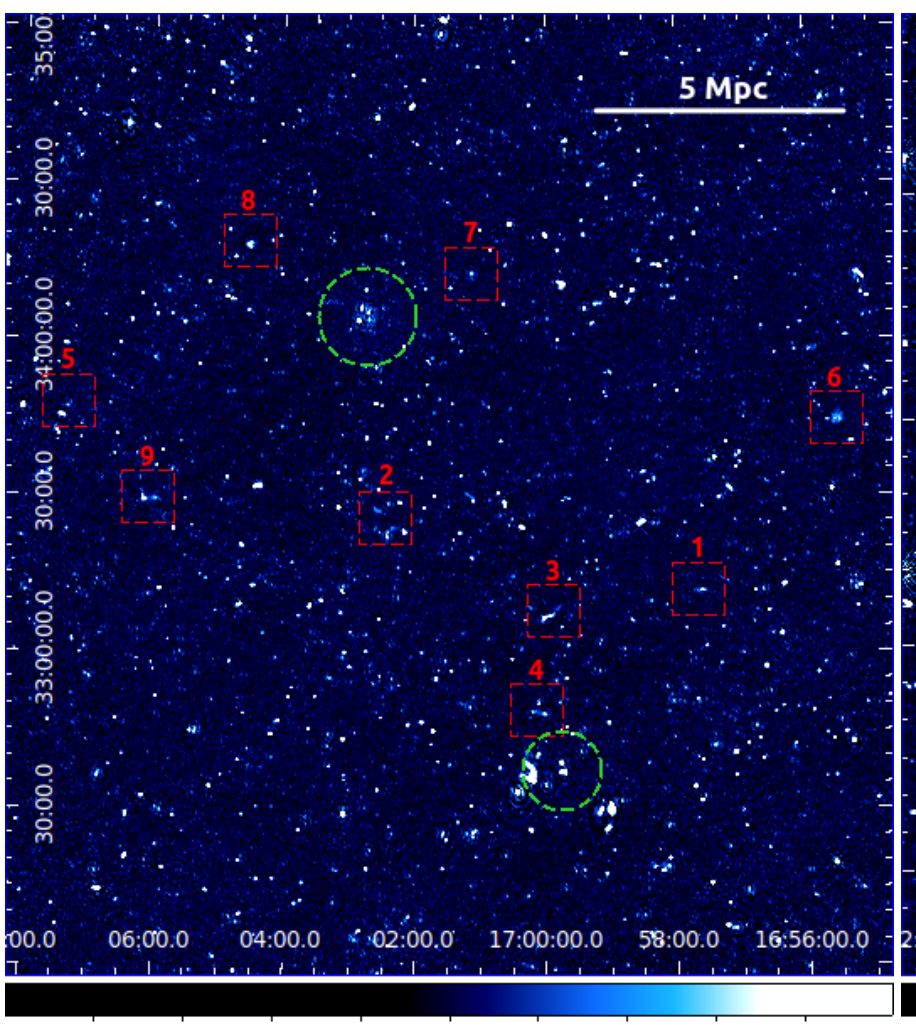

$-0.0032$

$$
-0.0016
$$

$-3.3 e-05$

0.0016

0.0032

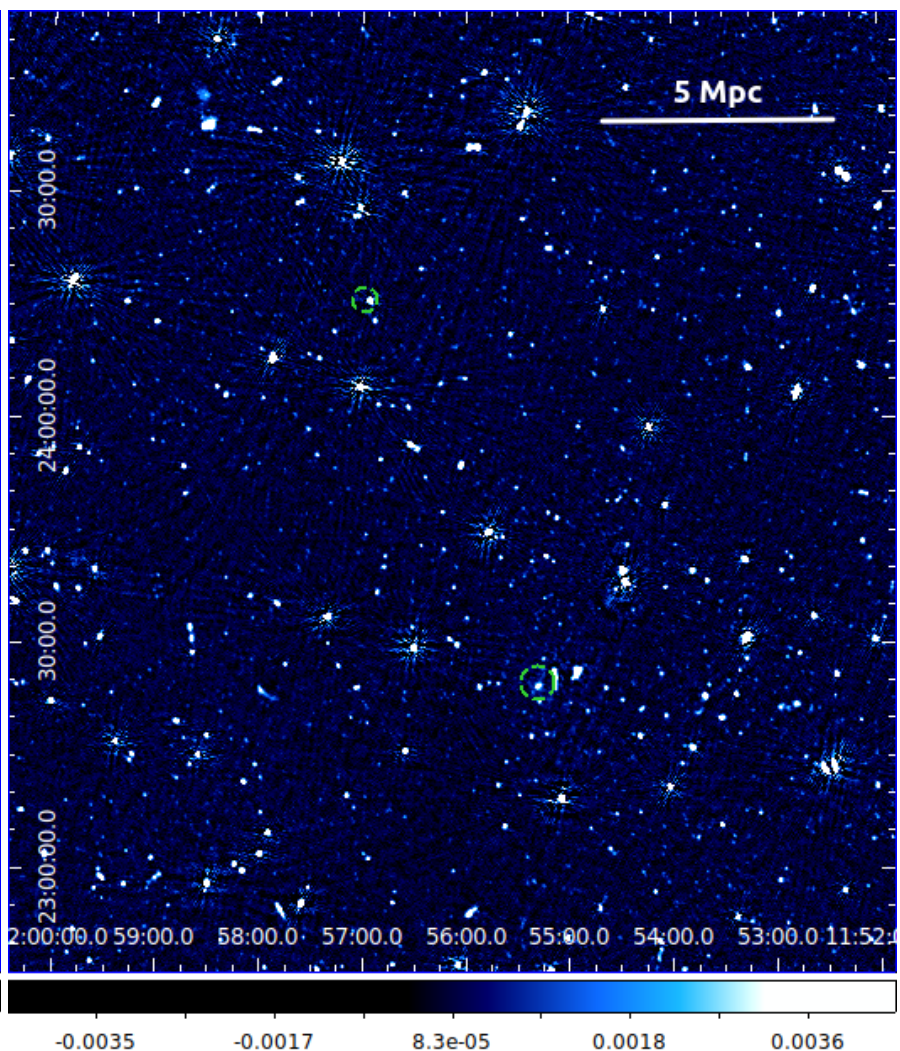

Fig. 2. LOFAR low-resolution (20") images at $150 \mathrm{MHz}$ of the cluster pairs RXC_J1659-J1702 (left panel) and RXC_J1155-J1156 (right panel). The dashed circles are centred on the clusters and have corresponding radius $R_{500}$. Magnified images of the red dashed boxes in the left panel are shown in Fig. 8. The $5 \mathrm{Mpc}$ unit at the redshift of the pairs $z=0.10,0.14$ is shown in the top right corner (left and right panels). The colour bars are in units of Jy beam ${ }^{-1}$.

namely $8 \mathrm{~h}$ on-source time between two $10 \mathrm{~min}$ scans on the flux density calibrator in the frequency range $120-168 \mathrm{MHz}$ using LOFAR in HBA_DUAL_INNER mode (see Shimwell et al. 2017, for details). A first calibration and imaging run was performed adopting the pipelines developed to analyse the LoTSS pointings (Shimwell et al. 2017, 2019); the aim was to correct for direction-independent and for direction-dependent effects exploiting the PREFACTOR (Williams et al. 2016; van Weeren et al. 2016; de Gasperin et al. 2019), KILLMS (Tasse 2014a,b; Smirnov \& Tasse 2015), and DDFACET (Tasse et al. 2018) software. In particular, we used the improved version of the direction-dependent data reduction pipeline $\left(\mathrm{v} 2.2^{2}\right)$, the same used for the forthcoming second LoTSS data release (DR2; Shimwell et al., in prep.) to produce images of the full LOFAR field of view at the central frequency of $144 \mathrm{MHz}$ at high (6") and low resolution (20", shown in Fig. 2) using a Briggs weighting scheme (robust $=-0.5$ ). We refer the reader to Tasse et al. (2021) for a thorough description of the steps performed by the pipeline. Using the sky models derived from the pipeline, we subtracted the sources from the $u v$-data in two different fashions: either we subtracted all sources (by means of their clean components) found in the high- and low-resolution maps or we subtracted all sources detected in the high-resolution image. The model components were determined during the high- and low-resolution images deconvolution making use of the PYthon Blob Detector and Source Finder (PYBDSF; Mohan \& Rafferty 2015). The subtraction of the model components was performed in the visibility domain, corrupting the model components by

https://github.com/mhardcastle/ddf-pipeline the direction-independent antenna gains obtained from the calibration. We then produced dirty images from the subtracted data. The images include the residual contribution to the surface brightness resulting from model approximation plus artefacts associated with imperfect model, and solutions ( $\operatorname{Im}_{\text {empty }}$ ) plus patches of faint extended emission in the case when only sources from the high-resolution model were subtracted $\left(\operatorname{Im}_{\text {diffuse }}\right)$. The subtracted (dirty) images $\operatorname{Im}_{\text {empty }}$ at low resolution $\left(20^{\prime \prime}\right)$ have an rms noise floor of $\sim 160$ and $240 \mu \mathrm{Jy}_{\text {beam }}{ }^{-1}$ for the two fields, respectively. The noise difference is consistent with the amount of flagged (i.e. discarded) data in the two observations.

\subsection{Cosmological simulations}

We extracted the simulated inter-cluster filaments from the suite of simulations of the cosmic web properties described in Vazza et al. (2019) performed with the cosmological MHD code $\mathrm{ENZO}^{3}$ (Bryan et al. 2014). The simulations consist of a comoving $100^{3} \mathrm{Mpc}^{3}$ box with a uniform grid of $2400^{3}$ cells (and $2400^{3}$ dark matter particles) with linear (comoving) resolution of $41.6 \mathrm{kpc}$ per cell and dark matter mass $m_{\mathrm{dm}}=8.62 \times 10^{6} M_{\odot}$ per dark matter particle. Magnetic fields are initialised at $z=$ 45 as a uniform background of $B_{0}=0.1 \mathrm{nG}$ and evolved at run-time using the MHD method of Dedner (Dedner et al. 2002). We note that a uniform initial magnetic field here corresponds to a scale-invariant spectrum in the models used for cosmic microwave background (CMB) analysis (Aghanim et al. 2019; Paoletti \& Finelli 2019). We also note that the run is

https://enzo-project.org 
non-radiative and does not include any treatment for star formation or feedback from active galactic nuclei (AGN). While to a first approximation these processes are not very relevant for the radio and X-ray properties of the peripheral regions of galaxy clusters and filaments (e.g. Gheller \& Vazza 2019), the effect of outflows from AGN and galaxies can mix metals and magnetic fields at least out to the virial radius of clusters (e.g. Biffi et al. 2018). Simulations by our group show that these effects are negligible on the radio emission on the scale of filaments (Vazza et al. 2017); however, only future and more refined simulations including effects related to galaxy formation in filaments at a much higher resolution, will be able to assess with more certainty whether the limits obtained from observations (such as those obtained in this work) can be straightforwardly related to a limit on primordial seed fields.

\subsection{Synchrotron emission model for cosmic shocks}

We produced synthetic maps of synchrotron radio emission assuming that diffusive shock acceleration (DSA; e.g. Kang et al. 2012 and references therein) accelerates a small fraction of thermal electrons swept by structure formation shocks up to relativistic energies, as in Vazza et al. (2019). We computed the radio emission from electrons in the downstream cooling region of shocks using the model of Hoeft \& Brüggen (2007), and based on the shocks identified in post-processing in the simulation. The total acceleration efficiency at shocks, $\xi_{\mathrm{e}}(\mathcal{M})$ (with $\mathcal{M}$ the Mach number) is assumed to be the combination of two variables: the kinetic energy flux dissipated onto the acceleration of cosmic rays, $\psi(\mathcal{M})$, and the fraction going into electron acceleration, $\xi_{\mathrm{e}}^{\prime}$, giving $\xi_{\mathrm{e}}(\mathcal{M})=\xi_{\mathrm{e}}^{\prime} \cdot \psi(\mathcal{M})$. Following Hoeft \& Brüggen (2007), the radio emission in the downstream of each shock is directly linked to the power-law energy distributions $N_{\gamma} \propto \gamma^{-\mathrm{p}}$ of electrons accelerated by the shock front during a cooling time through the integrated radio spectrum of $I(v) \propto v^{-\mathrm{s}}$, where $s=(p-1) / 2+1 / 2$, with $p=2\left(\mathcal{M}^{2}+\right.$ $1) /\left(\mathcal{M}^{2}-1\right)$. With this approach and for the range of $\mathcal{M} \gg 5$ shocks usually found within and around simulated filaments, and for the $\leq \mu \mathrm{G}$ magnetic fields in filaments (e.g. Vazza et al. 2017), the radio emission thus scales as $I(v) \propto \xi_{\mathrm{e}} B^{2} v^{-2}$. The baseline model used in this work assumes $\xi_{\mathrm{e}}=10^{-2}$, which is in line with DSA expectations for the maximum acceleration efficiency of relativistic electrons by strong shocks (e.g. Hoeft \& Brüggen 2007; Kang et al. 2012; Bykov et al. 2019a) and is also compatible with the modelling of supernova remnants (e.g. Uchiyama et al. 2007; Bykov et al. 2019b). However, in typically weak shocks $(\mathcal{M} \leq 4)$ leading to radio relics in galaxy clusters (e.g. van Weeren et al. 2019) the acceleration efficiencies implied by the observed relic radio fluxes can be much larger ( $\xi_{\mathrm{e}} \sim 0.1-1$; see e.g. Stuardi et al. 2019; Botteon et al. 2020b), thus making our maximum value of $\xi_{\mathrm{e}}=10^{-2}$ a conservative one. We note, however, that very recent particle-in-cell (PIC) simulations (albeit in 1D and with some limiting assumptions) derive a $\sim 5 \%$ electron acceleration efficiency by strong shocks (Xu et al. 2020). For the remainder of the paper it should be kept in mind that our limits on $B_{10 \mathrm{Mpc}}$ must be accordingly rescaled if a different value of $\xi_{\mathrm{e}}$ is adopted.

Figure 3 gives an example of filaments connecting a massive cluster to other groups in its surrounding, in an ENZO cosmological simulation. A small but significant fraction of radio emission from shocks running on filaments connecting some of the pairs (e.g. M1-M2, M1-M4, and M1-M6 in Fig. 3) is above the detection threshold in LOFAR-HBA for our baseline model. In all cases the detectable emission comes from relatively small and

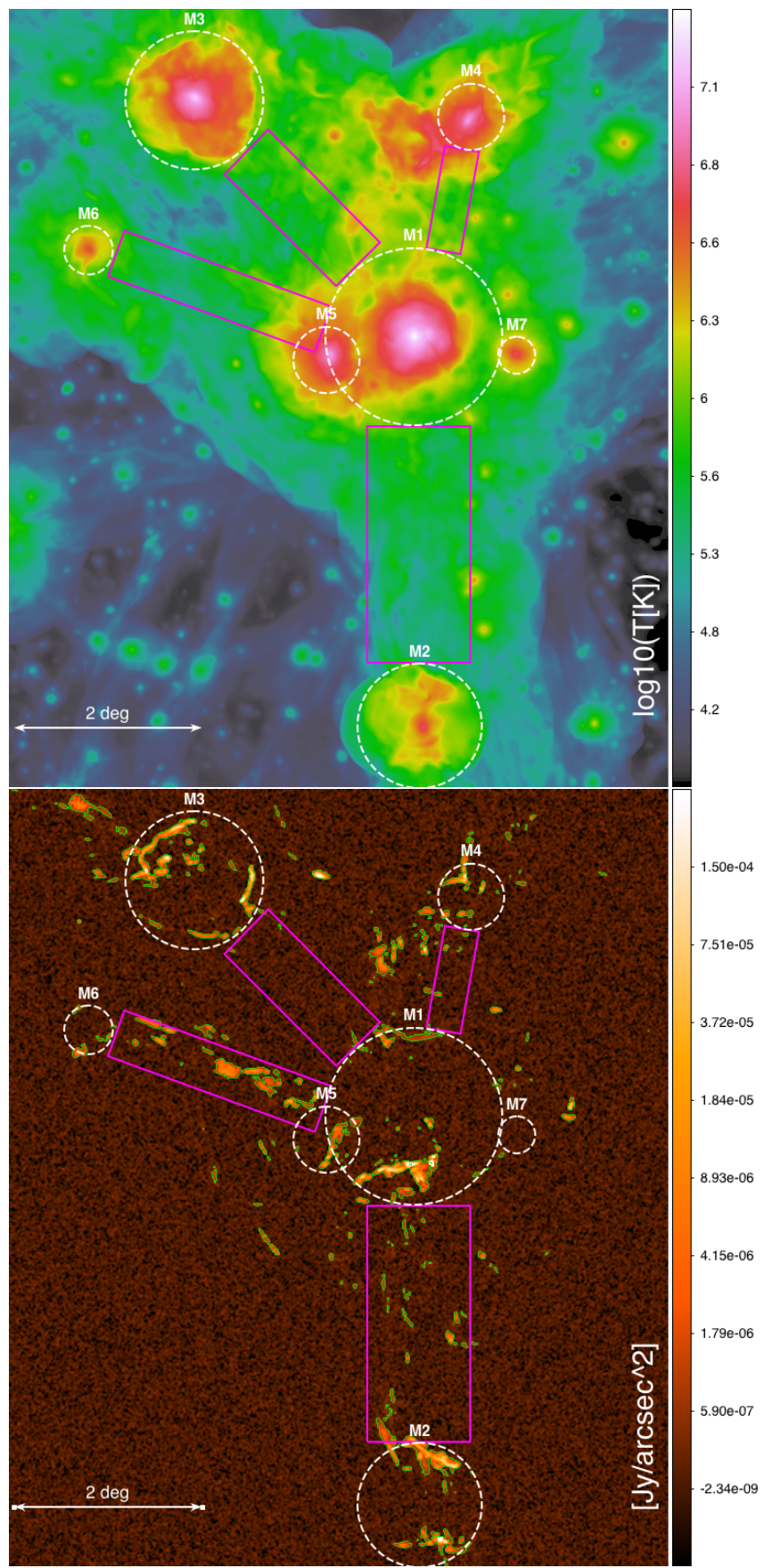

Fig. 3. Example of mean gas temperature (top) and mock LOFAR-HBA observation (bottom, $v=140 \mathrm{MHz}, 25^{\prime \prime}$ resolution, $250 \mu \mathrm{Jy}^{\text {beam }}{ }^{-1}$ noised added) from the ENZO simulation. The circles indicate the virial region of clusters; the magenta rectangles show the filaments. The detectable emission $(\geq 3 \sigma)$ is shown as green contours. The cluster field is placed at $z=0.1$.

localised patches, extended a few $\simeq 10^{\prime}-20^{\prime}$ at most, with irregular shapes. Detecting the radio signal from cosmic filaments is indeed made challenging by the fact that the detectable fraction is just the tip of the iceberg of the wider 'radio cosmic web', which often makes a morphological classification of the emission ambiguous. Advanced Deep Learning techniques have been proposed for the detection of the cosmic web in future radio surveys (Gheller et al. 2018). In the following section we use this model to constrain the amplitude of the $\xi_{\mathrm{e}} B^{2}$ combination based on our real LOFAR observations. 

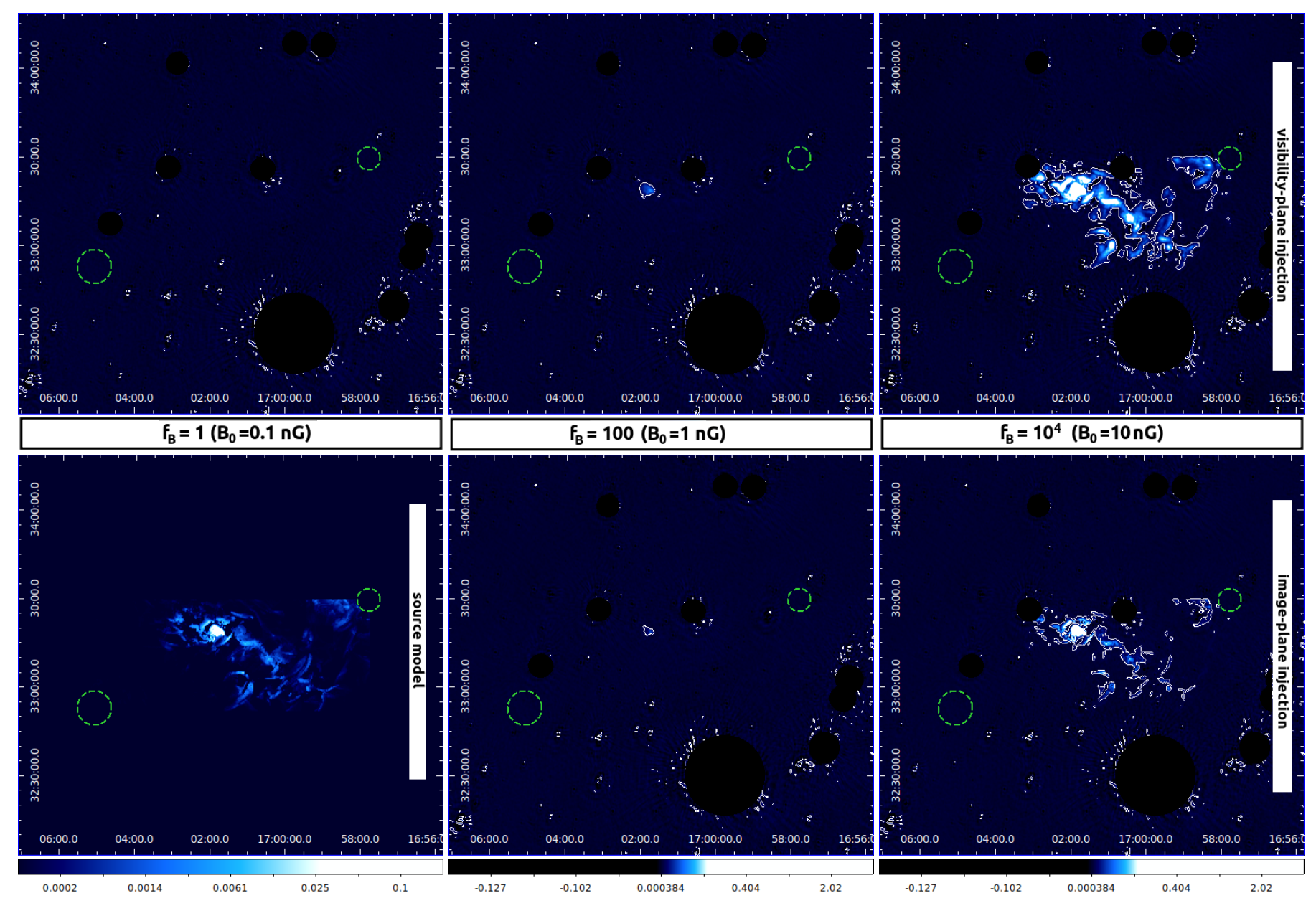

Fig. 4. Example of source injection. The model of diffuse emission between a pair of simulated galaxy clusters (masked within their $R_{200}$; green dashed circles) found in the simulation with $B_{0}=0.1 \mathrm{nG}$ (lower left panel) is multiplied by a factor $f_{B}=1,10^{2}, 10^{4}$ imaged and masked after being injected into the source-subtracted sky visibilities (upper left, upper central, and upper right panels, respectively), or it is injected through the image-plane (lower centre and lower right panels for $f_{B}=10^{2}$ and $10^{4}$, respectively). The image with $f_{B}=1$ in the upper left panel, due to the very low brightness of the model with $B_{0}=0.1 \mathrm{nG}$, is equal to the source-subtracted sky image $\operatorname{Im}_{\text {empty }}$, where the only features are the residuals from the source subtraction process that fall outside the masks. The white contours are set to 5 times the rms value in $\operatorname{Im}_{\text {empty }}$.

\subsection{Generation of a mock catalogue of inter-cluster filaments}

For each observed pair of clusters we selected simulated pairs holding individual cluster masses $M_{500}>10^{13} M_{\odot}$ and linear (comoving) and projected angular distance of clusters in the pair within $20 \%$ deviation from the values of the observed pair (see the last and second-last columns in Table 1). We obtained 171 simulated cluster pairs selected for RXC_J1659-J1702 and 139 pairs for RXC_J1155-J1156. The cluster pairs selected above mirror the separation selection criteria of our observations, but include less massive clusters that may not involve a physical connection within one pair. We thus also analysed a subsample of high-mass clusters $\left(M_{500}>3 \times 10^{13} M_{\odot}\right)$ for which a physical connection and the presence of a inter-cluster filament was verified manually and through a high temperature cut $T_{\text {WHIM }}>10^{5} \mathrm{~K}$ of the WHIM within the inter-cluster filament. We refer to this subsample as best.

Using a simulated cluster pair, a box was drawn and extracted along the direction connecting the pair. The box was rescaled to match the angular scale and comoving transverse distance indicated by the pixel size and redshifts of the observed clusters, and the intensity of synchrotron emission was scaled to match its luminosity distance by conserving the total power (see the lower left panel in Fig. 4 for an example).
The flux density was also multiplied by a constant factor $f_{B} \equiv\left[B_{0} /(0.1 \mathrm{nG})\right]^{2}$. Under the assumption that the amplification of the magnetic field into the simulated filaments is affected by negligible small-scale dynamo (Ryu et al. 2008) and that it is thus mainly driven by the adiabatic compression of the magnetic field lines following from flux freezing ${ }^{4}$ into the plasma condensing during structure formation (Vazza et al. 2017), the magnetic field $B$ in the simulation is scalable with respect to $B_{0}$ and in turn the synchrotron emissivity is scalable with respect to $f_{B} \propto B_{0}^{2}$ (assuming a constant $\xi_{\mathrm{e}}=10^{-2}$ ).

\subsection{Injection of model radio emission into real LOFAR images}

The rescaled simulated image of each mock pair of galaxy clusters was injected into the source-subtracted measurement set (MS), following the procedure sketched in Fig. 1. In detail, a Fourier transform was performed on each rescaled image; the image was then written into the MS and finally added to the visibilities of the source-subtracted sky using WSCLEAN (Offringa et al. 2014). We note that this injection does not take

4 The magnetic flux through a closed loop C enclosing the surface $S$ is simply $\Phi_{B}=\int_{\mathrm{S}} \boldsymbol{B} \cdot \mathrm{d} \boldsymbol{S}$, valid for ideal plasma conditions. 
into account direction-dependent effects that may act on the MS radio data. With the same software the resulting data set was imaged and deconvolved with a $20^{\prime \prime} u v$-taper and synthesised beam, and Briggs weighting scheme (Briggs 1995) with robust $=-0.25$ and 2000 minor cycles (see Fig. 4 for output examples). For realistic values of the normalisation parameter $f_{B}$, the detectable emission is fragmented into small and sparse patches, associated with shocks internal to filaments. Therefore, we resort to statistical methods to assess the likelihood of each mock image to be compatible with our observed LOFAR fields. We compute the integral of the image power spectrum $P_{\mathrm{S}} \equiv \log _{10}\left(\int_{k_{\min }}^{k_{\max }} P\left(k^{\prime}\right) \mathrm{d} k^{\prime}\right)$, where $P\left(k^{\prime}\right)$ is the power spectrum and $k_{\min }$ and $k_{\max }$ are determined by the image and beam size, respectively (see Fig. 9 for an example). The sky model subtraction can leave bright residual artefacts depending on the goodness of the model used. These residuals can be as bright as $\sim 0.1 \mathrm{Jy} \mathrm{beam}^{-1}$ around point-like sources and they may dominate the integral of the image power spectrum $P_{\mathrm{S}}$. A zeropadding mask was manually generated for each pair of clusters in order to exclude those artefact from the computation of $P_{\mathrm{S}}$ in all images.

\section{Results}

From the cumulative probability distributions of the statistic $P_{\mathrm{S}}$ resulting from all the source injections, we can assess how likely it is for a model to provide an expected value smaller than the one recovered from the observations. Using the image $\operatorname{Im}_{\text {empty }}$, a $2.5 \mathrm{deg} \times 2.5 \mathrm{deg}$ square centred on the axis of the cluster pair, from which all the sources (point-like plus extended) have been subtracted, we compute $\tilde{P}_{\mathrm{S}} \equiv P_{\mathrm{S}}\left(\operatorname{Im}_{\text {empty }}\right)$. The parameter $\tilde{P}_{\mathrm{S}}$ corresponds to the total power in $\operatorname{Im}_{\text {empty }}$ distributed over all scales from twice the beam size $\left(k_{\min }\right)$ up to half the image size $\left(k_{\max }\right)$. All images resulting from injection thus have $P_{\mathrm{S}}$ equal to or larger than the value computed for $\operatorname{Im}_{\text {empty }}$ (red dotted lines in Fig. 5). The statistic $P_{S}$ resulting from the image in which diffuse emission has not been subtracted $\operatorname{Im}_{\text {diffuse }}$ are indicated by the blue dashed vertical lines in Fig. 5. Totals of 6 and $15 \mathrm{mJy}$ of diffuse emission have been found in $\operatorname{Im}_{\text {diffuse }}$ in excess of $\mathrm{Im}_{\text {empty }}$ for RXC_J1659-J1702 and RXC_J1155-J1156, respectively. We outline the probabilities for the different models in Table 2, which also lists the results from the injection performed in the image plane found in general to produce different probabilities of non-detection with respect to injection through the $u v w$-plane. We discuss this alternative method in Sect. 4.

The overall probability of a magnetic field model is simply the product of the probabilities (of the model to produce lower statistics) of the two cluster pairs, since the experiments were run independently on each pair. We compute these probabilities in Table 2 (see 'all', bottom panel). A model is more likely to be discarded when its probability of having a smaller $P_{\mathrm{S}}$ than in our LOFAR observations is either very small or very high.

Our main results can be summarised in the following way. The primordial scenario with a seed magnetic field of $B_{0} \simeq$ $10 \mathrm{nG}$ has a small probability $P\left(<P_{\mathrm{S}}\right) \simeq 0.05$ of explaining the small power excess in our observation of the RXC_J1659-J1702 and RXC_J1155-J1156 pairs, and we thus reject it with a confidence level (CL) of $>95 \%$. The models with a lower seed magnetic fields $B_{0}<10 \mathrm{nG}$ yield non-negligible probabilities $(\geq 0.1)$ of producing a statistic equal to (or smaller than) the one observed. By tightening the constraints on individual cluster masses and on the presence of an inter-cluster filament connecting the clusters the model with $B_{0}=10 \mathrm{nG}$ can be rejected with
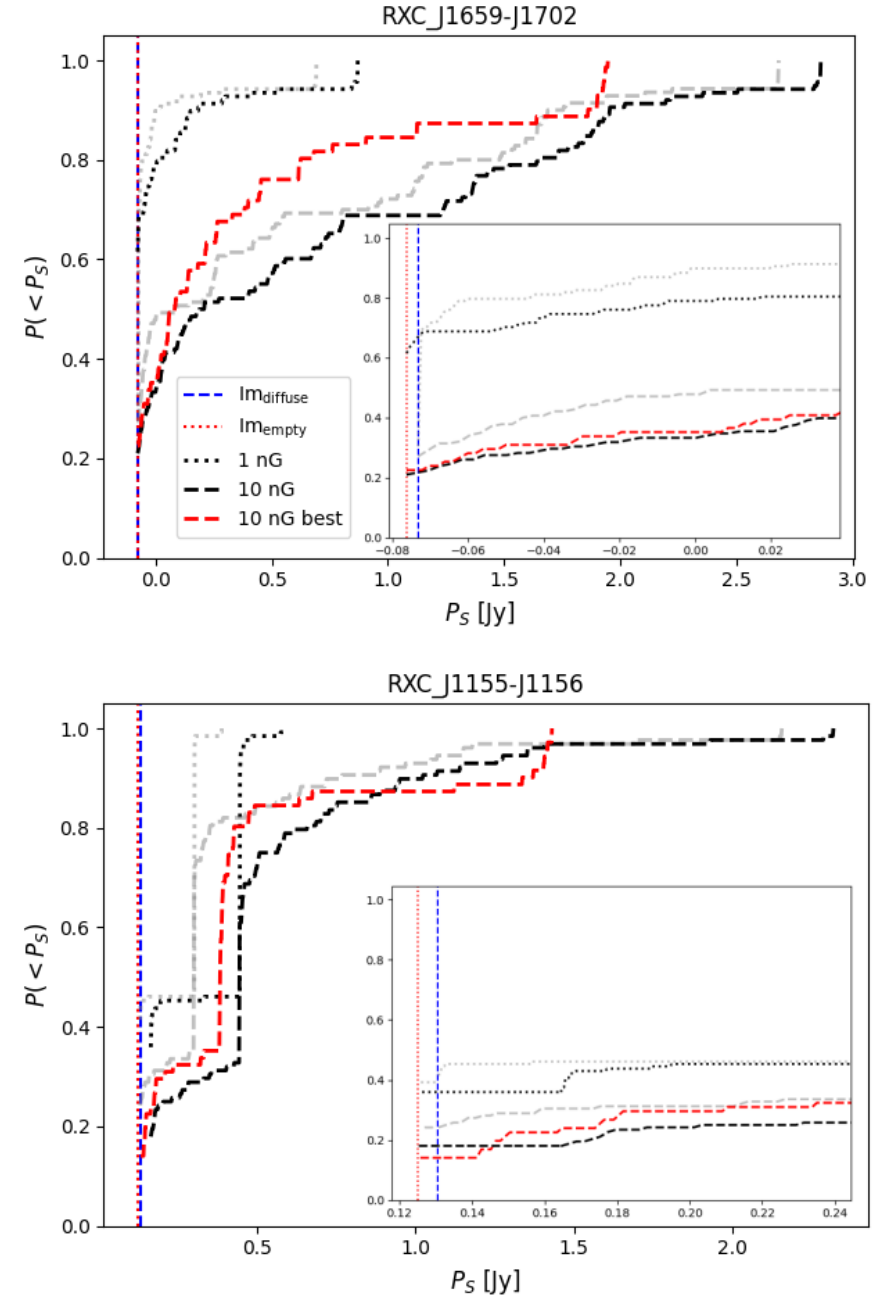

Fig. 5. Probability distributions of finding statistics $P_{\mathrm{S}}$ smaller than the values set by the cluster pair RXC_J1659-J1702 (upper panel) and RXC_J1155-J1156 (lower panel) for scenarios with $B_{0}$ as labelled. The vertical lines show the $P_{\mathrm{S}}$ values computed without any injection from $\mathrm{Im}_{\text {empty }}$ (red dotted) or $\mathrm{Im}_{\text {diffuse }}$ (blue dashed). The black and grey lines show results for source injection performed respectively in the visibility and image domains. The insets show a zoom-in on the bins where $P\left(<P_{\mathrm{S}}\right) \equiv P\left(<\tilde{P}_{\mathrm{S}}\right)$.

even higher confidence, CL $>97 \%$. If any of the patches of diffuse emission observed is produced by shocks in the WHIM, then the $B_{0}=0.1 \mathrm{nG}$ model is highly disfavoured as it is basically unable to produce any detectable emission (i.e. the probability in Table 2 that this model produces less diffuse emission than found in $\operatorname{Im}_{\text {empty }}$ is always $\approx 1$; these values are not plotted in Fig. 5). Although we do not reject this scenario, we consider it implausible (see Sect. 4).

\section{Discussion}

From the original simulation holding $B_{0}=0.1 \mathrm{nG}$, we extract the probability distribution functions (PDF) of the magnetic field values $B_{10 \mathrm{Mpc}}$ across the mock filaments selected according to the properties of the observed cluster pairs. We plot the resulting PDF $\left(\log B_{10 \mathrm{Mpc}}\right)$ in Fig. 6. Given the expected lack of dynamo amplification in the WHIM, the magnetic field distributions PDF $\left(\log B_{10 \mathrm{Mpc}}\right)$ corresponding to the other $B_{0}$ models can easily be rescaled linearly with the input seed field. We find a skewed 
Table 2. Probabilities of obtaining a statistic $P_{\mathrm{S}}$ lower than the one observed in $\mathrm{Im}_{\text {empty }}$ and $\operatorname{Im}_{\text {diffuse }}$, computed from source injection performed in the $u v w$-plane and in the image-plane.

\begin{tabular}{|c|c|c|c|c|c|c|}
\hline \multirow[t]{2}{*}{ Name } & \multirow[t]{2}{*}{$f_{B}$} & \multirow{2}{*}{$\begin{array}{c}B_{0} \\
{[\mathrm{nG}]} \\
\end{array}$} & \multicolumn{2}{|c|}{$u v w$-plane } & \multicolumn{2}{|c|}{ Image-plane } \\
\hline & & & Im $_{\text {empty }}$ & $\operatorname{Im}_{\text {diffuse }}$ & $\operatorname{Im}_{\text {empty }}$ & $\operatorname{Im}_{\text {diffuse }}$ \\
\hline \multirow[t]{3}{*}{ RXC_J1659-J1702 } & 1 & 0.1 & 1 & 1 & 1 & 1 \\
\hline & $10^{2}$ & 1 & 0.62 & 0.67 & 0.68 & 0.68 \\
\hline & $10^{4}$ & 10 & 0.21 & 0.21 & 0.27 & 0.27 \\
\hline *Best & & & 0.23 & 0.23 & 0.24 & 0.24 \\
\hline \multirow[t]{3}{*}{ RXC_J1155-J1156 } & 1 & 0.1 & 1 & 1 & 1 & 1 \\
\hline & $10^{2}$ & 1 & 0.36 & 0.36 & 0.42 & 0.42 \\
\hline & $10^{4}$ & 10 & 0.18 & 0.18 & 0.24 & 0.24 \\
\hline *Best & & & 0.14 & 0.14 & 0.18 & 0.18 \\
\hline \multirow[t]{3}{*}{ All } & 1 & 0.1 & 1 & 1 & 1 & 1 \\
\hline & $10^{2}$ & 1 & 0.22 & 0.24 & 0.29 & 0.29 \\
\hline & $10^{4}$ & 10 & 0.04 & 0.04 & 0.06 & 0.06 \\
\hline *Best & & & 0.02 & 0.03 & 0.04 & 0.04 \\
\hline
\end{tabular}

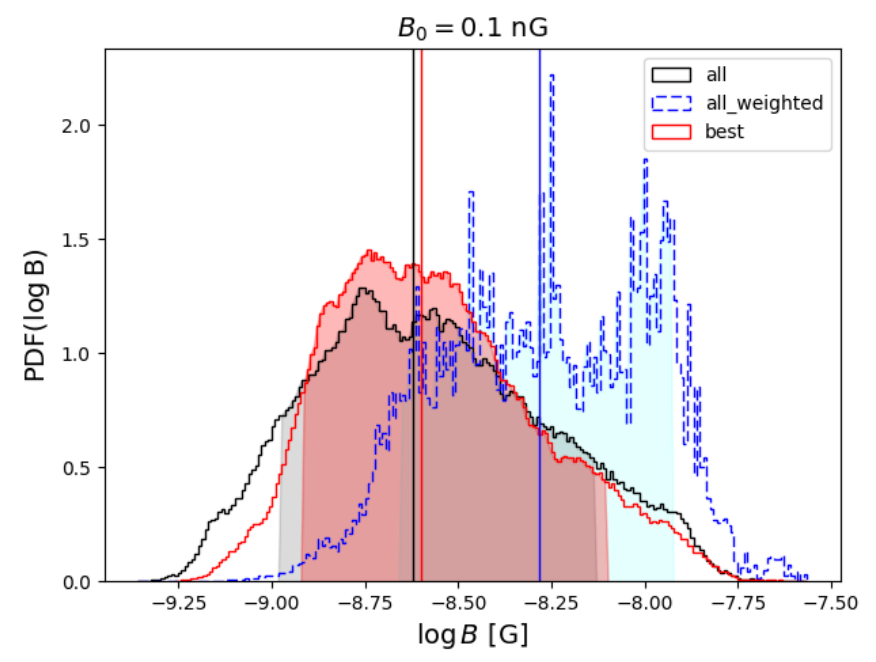

Fig. 6. PDFs of the $\log B_{10 \mathrm{Mpc}}$ field across all the simulated filaments in the $B_{0}=0.1 \mathrm{nG}$ model, for all the pairs in the mock sample (black line) and for the best subsample (red line). The dashed blue lines show the $\log B_{10 \mathrm{Mpc}}$ distribution from all pairs weighted over the pixel emissivity. The filled hatched areas encompass the 10-90 percentile ranges. The vertical solid lines show the median of the distributions.

distribution encompassing $B_{10 \mathrm{Mpc}}=1.0-7.4 \mathrm{nG}$ values $(90 \%$ confidence range) with median $B_{10 \mathrm{Mpc}}=2.5 \mathrm{nG}$ (equivalent to $\left.\log B_{10 \mathrm{Mpc}}=-8.6\right)$ for the full sample. We note that the value of the magnetic field that produces the simulated synchrotron emission is in the high part of the $B_{10 \mathrm{Mpc}}$ distribution, as can be seen from the emission-weighted $B_{10 \mathrm{Mpc}}$ distribution in Fig. 6. We also give in Fig. 7 the average profiles of mass-weighted magnetic field strength for all simulated filaments extracted with the procedure above, for the two cluster pairs. On average, the profile of magnetic field is very uniform across $10-20 \mathrm{Mpc}$, with an average magnetic field along the line of sight for these objects of $\sim 2-3 \mathrm{nG}$ and a tail of rare and massive filaments that can reach $\sim 10 \mathrm{nG}$ (Fig. 7). We find that the magnetic field along the direction connecting the clusters is enhanced with respect to the volume-filling values. The enhancement is significant regardless of whether the gas reaches temperatures typical of filaments $\left(T>10^{5} \mathrm{~K}\right)$, but the enhancement is greater for higher temperatures, as expected from compression of the magnetic field lines during the structures growth (e.g. Gheller et al. 2016).
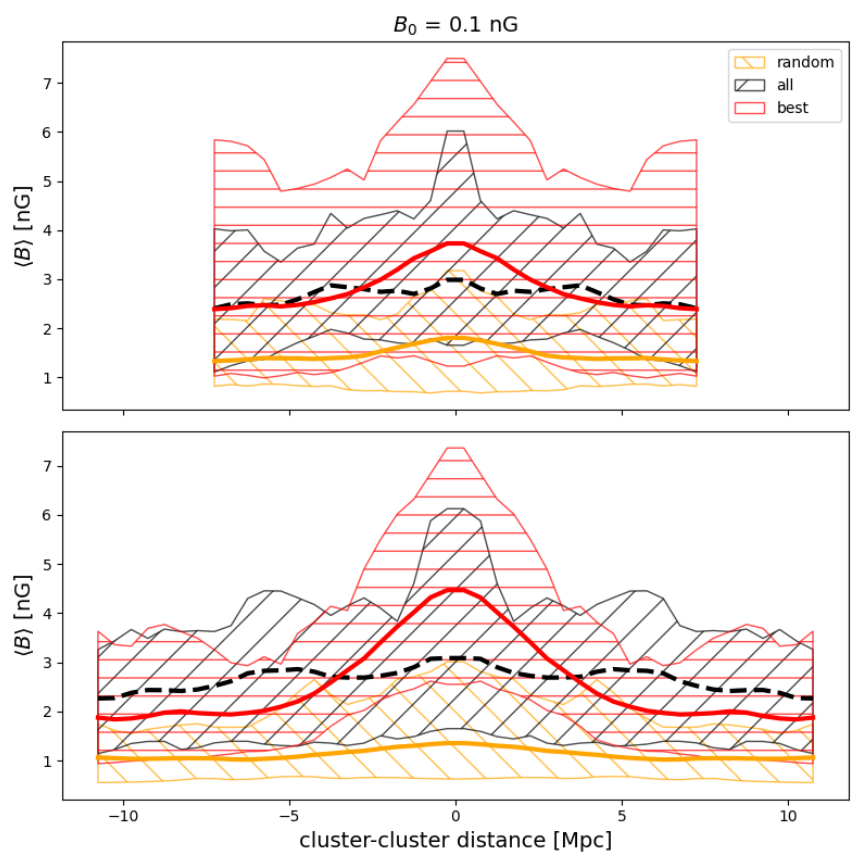

Fig. 7. Magnetic field profile along the cluster-to-cluster distance. Upper panel: average profile of mass-weighted magnetic field strength resulting from the $B_{0}=0.1 \mathrm{nG}$ model for all inter-cluster filaments (black), extracted to resemble the cluster pair RXC_J1155-J1156; for the best subsample (red); and for a sample of images sampling an equal linear size $(15 \mathrm{Mpc})$ extracted at random locations in the simulation (orange). The solid lines give the median values of the samples, while the filled areas encompass the 10-90th percentiles of the distributions. Lower panel: same plot, but for the mock sample extracted for RXC_J1659-J1702.

To interpret the results provided in Fig. 5 and Table 2, we postulate three different assumptions that exploit the different types of source-subtraction performed in the analysis, which can be used to derive different priors from our data (vertical lines in Fig. 5):

I: None of the residual diffuse emission after the point-like source subtraction $\left(\mathrm{Im}_{\mathrm{empty}}\right)$ is produced by the shocked cosmic web;

II: All of the residual diffuse emission in excess of $\operatorname{Im}_{\text {empty }}$ (i.e. $\left.\operatorname{Im}_{\text {diffuse }}\right)$ is produced by the shocked cosmic web; 
III: At least some of the excess diffuse emission present in Im $_{\text {diffuse }}$ comes from the cosmic web.

We analyse implications of the three assumptions separately in the following paragraphs.

\subsection{Hypothesis I}

Provided that we can fix the $\xi_{\text {e }}$ acceleration efficiency at strong shocks $\left(\xi_{\mathrm{e}} \approx 10^{-2}\right)$, the assumption that none of the observed emission comes from cosmological shocks (I) produces, in principle, tighter constraints on $B_{10 \mathrm{Mpc}}$ (and $B_{0}$ ) since $P\left(P_{\mathrm{S}}\left(\operatorname{Im}_{\text {empty }}\right)\right) \leq P\left(P_{\mathrm{S}}\left(\operatorname{Im}_{\text {diffuse }}\right)\right)$ always. In practice, the constraints are just slightly tighter due to the small amount of diffuse emission found in $\operatorname{Im}_{\text {diffuse }}$ with respect to $\operatorname{Im}_{\text {empty }}$. Thus, under the first hypothesis that we did not observe the cosmic web emission, by scaling the $B_{10 \mathrm{Mpc}}$ distribution obtained from $B_{0}=0.1 \mathrm{nG}$ to match the $B_{0}=10 \mathrm{nG}$ model (i.e. a factor $\times 100$ ) we infer an upper limit to the current median IGMF in filaments of $B<0.25 \mu \mathrm{G}$ with $95 \%$ confidence (the same confidence level that applies to the rejection of $B_{0} \geq 10 \mathrm{nG}$ models of the primordial magnetic field scenario). By considering the best subsample of cluster pairs with higher masses and connected by an intercluster filament, we can further improve the constraints on $B$ by rejecting the $B_{0}=10 \mathrm{nG}$ model with a higher CL $>97 \%$.

Not all pairs of clusters are physically connected by a cosmic filaments, and in the absence of a detection in other wavelengths (via Sunyaev-Zeldovich or X-ray analysis; e.g. Govoni et al. 2019) one needs to resort to linking probabilities as a function of distance, which can be derived from cosmological simulations. For example, early dark matter-only simulations estimated that $\sim 80 \%$ of pairs in the same mass range as our clusters, and separated by $\sim 15 \mathrm{Mpc} h^{-1}$, are physically connected by filaments (Colberg et al. 2005). With more recent and resolved simulations, also including gas physics, we can revise this number and tailor it to the exact mass difference and separation of our LOFAR pairs. Using the algorithm outlined in Banfi et al. (2021), we reconstructed the network of filaments connecting halos in our simulation by checking for the actual presence of a matter bridge between pairs of clusters with a 25 or a $15 \mathrm{Mpc}$ separation. This allows us to associate a probability for the presence of a filament with each LOFAR observation, through the ratio of the number of physical filaments in the simulation (best sample) to the total pairs of galaxy clusters found at a given distance. This results in a $35 \%$ and $65 \%$ probability of having a filament between clusters at 25 and $15 \mathrm{Mpc}$ separation, respectively. It should be noted that even in the case without an actual gas connection between clusters, the region in between is far from empty, because massive clusters at a relatively short separation are also indicators of a large cosmic overdensity, which is often associated with the presence of other filaments or threads of the cosmic web along the line of sight, which may account for a non-negligible radio emission. This still allows us to exclude $B_{0}>10 \mathrm{nG}$ with high enough confidence. So, the absence of an actual filament does not dramatically affect the validity of the limits inferred from the full sample in this work.

\subsection{Hypothesis II}

The assumption that the excess diffuse emission present in $\mathrm{Im}_{\text {diffuse }}$ with respect to $\mathrm{Im}_{\text {empty }}$ is entirely due to shocked plasma of the WHIM can be readily tested by looking in detail at the diffuse emission patches that have been detected. In Fig. 8 we present close-up clippings of the diffuse patches found close to the pair RXC_J1659-J1702, taken from the low-resolution
LOFAR images (before source subtraction). They are meant to help in assessing the nature of some of the diffuse emission (dashed red circles in Fig. 8). We also give the position of sources already known from the VLA Faint Images of the Radio Sky at Twenty-centimeters (FIRST) survey (Becker et al. 1995). Most of the diffuse emission is plausibly linked to the lobes of radiogalaxies already detected at higher frequencies. Panel 7 shows what looks like either a radio lobe or an artefact linked to a low-frequency point source. Panels 2, 5, and 6 instead show diffuse emission that is not obviously linked to radio galaxies or to deconvolution artefacts. However, sources 5 and 6 are found (by looking at their coordinates) to be distant from the axis connecting the clusters, albeit within the imaged portion of the sky around the pair (see also Fig. 2). This makes their physical connection to the putative inter-cluster filament unlikely, even if the shocked cosmic web is expected to fill the space between the clusters in a non-trivial way, as shown in Fig. 3. Furthermore, the point-like source embedded in the diffuse emission in panel 6 is also found to be at a different redshift with respect to the cluster pair. For the above reasons, these patches can hardly be used in the comparison with the simulated inter-cluster filaments. The diffuse patches in panel 2 instead embed optical galaxies with redshift $z=0.087,0.093$, consistent with the cluster pair $z=0.095-0.101$; however, they are likely dying faint radio lobes with no FIRST counterpart. Interestingly, the positions of Sloan Digital Sky Survey (SDSS) sources (orange plus signs) in the panels of Fig. 8 seem to cluster close to the diffuse radio emission. This trend is actually expected if the radio signal is caused by radio galaxies and lobes, which tend to be found in clusters or groups of galaxies (Prestage \& Peacock 1988; Allington-Smith et al. 1993; Zirbel 1997; Wing \& Blanton 2011). This behaviour additionally disfavours the link between the excess diffuse emission and the cosmic web. All in all, since there is no easy way to cross-check all the different patches, we still compute the statistics for the most conservative scenario by assuming that the level of observed diffuse emission in excess of $\mathrm{Im}_{\text {empty }}$ is entirely due to the cosmic web. In this case the level of confidence associated with the rejection of the same models loosens. However, the models rejected by casting hypothesis II are the same ones resulting from hypothesis I, though at a slightly lower or even equal CL; for example, for the $B_{0}=10 \mathrm{nG}$ best model the CL (for its rejection) decreases from $98 \%$ to $97 \%$, while for the full sample it remains unchanged. Furthermore, since hypothesis II has already been falsified by the examples described above and shown in Fig. 8, the consensus for hypothesis I is strengthened, and we thus refer to it in order to draw our conclusions.

\subsection{Hypothesis III}

For completeness, we present an additional and interesting way of interpreting our data with respect to the simulation results, which is complementary to hypothesis I. We assume that at least some of the diffuse emission in excess of Im $_{\text {empty }}$ comes from the cosmic web. The associated probability is then trivially $P\left(>P_{\mathrm{S}}\right)=1-P\left(<P_{\mathrm{S}}\right)$. In this case we are not interested in the level of diffuse emission in $\operatorname{Im}_{\text {diffuse }}$ since we want to produce at least the one in $\mathrm{Im}_{\text {empty }}$. This scenario, though disfavoured, cannot be discarded a priori since this would imply checking (e.g. through cross-correlations) all the different patches of diffuse emission in $\operatorname{Im}_{\text {diffuse }}$ and proving that all of them are not connected to the emission from the Cosmic Web; it is then instructive to inspect its implications. Under the assumption that we did see the cosmic web emission at least in part, then the 

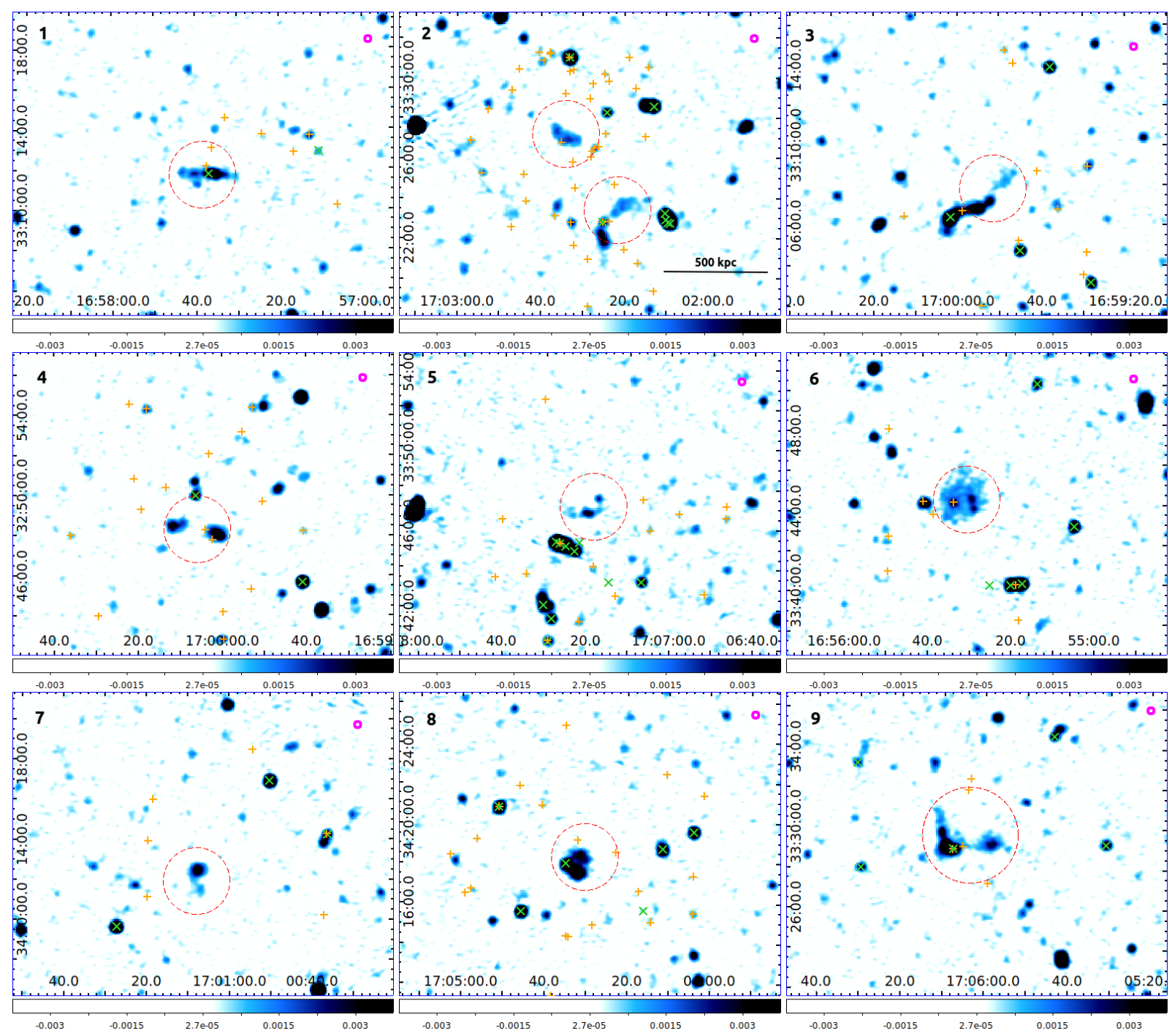

Fig. 8. Zoomed-in low-resolution sky images $\left(20^{\prime \prime}\right.$, magenta solid circles) centred over the patches of diffuse emission found in $\operatorname{Im}_{\text {diffuse }}$ of RXC_J1659-J1702 (dashed red circles). The green crosses $(\times)$ show the position of FIRST sources. The orange plus signs (+) show the position of SDSS galaxies with known spectroscopic redshift. Panels are numbered from 1 to 9 going from left to right and top to bottom. $1^{\prime \prime}=1.84 \mathrm{kpc}$ at $z=0.10$; the $500 \mathrm{kpc}$ scale is shown in panel 2 for comparison. The colour bars are in units of Jy beam ${ }^{-1}$.

$B_{0}=0.1 \mathrm{nG}$ model is ruled out with high confidence $(\geq 99 \%)$ since it is not able to produce any observable emission brighter than the noise level of our LOFAR observation. In this scenario $B_{0}>0.1 \mathrm{nG}$ can be set as a lower limit to the primordial magnetic field intensity, and in turn $B>2 \mathrm{nG}$ as the median value for the magnetic field into filaments today.

\subsection{Visibility versus image plane injection}

While checking that the source injection procedure (presented in Sect. 2 and sketched in Fig. 1) is actually needed in order to derive robust limits on $B_{10 \mathrm{Mpc}}$ and $B_{0}$ we also demonstrate that the method is essential to interpret observations in detail by means of the outcome of simulations when dealing with radio data. In this respect, we produced the same statistic $P_{\mathrm{S}}$ for the simulations directly added to the residual image $\operatorname{Im}_{\mathrm{empty}}$ in terms of a simple image sum, rather than following the central $\mathrm{FFT}+$ write + sum procedure involving visibilities. This procedure is much simpler and faster (shortening the computing time by a factor of $\sim 600$ ). In Fig. 9 we plot the power spectra resulting from the injection in the RXC_J1659-J1702 field of one source as an example (images of the same source are shown in Fig. 4) in order to inspect the differences between the injection through the $u v w$-plane (black lines) and the image-plane (grey lines) for the different $B_{0}$ models. As can be seen by comparing the black and grey lines, when the injection is performed within the imageplane the level of simulated emission on large scales is generally underestimated. As a consequence the models are consistent with the data with different probabilities up to $\pm 30 \% P\left(<P_{\mathrm{S}}\right)$ (see the values in Table 2). We interpret the difference in the results as being due to the lack of model convolution with the instrument's point spread function (PSF). In addition, the lack of convolution of the emission with a visibility weighting scheme able to maximise the evidence for extended diffuse emission into the data may also play a similar role. As long as an upper cut on the scales of the emission (corresponding to a lower bound on the baseline length in radio interferometers) is taken into account, and detailed power spectrum information does not constitute the largest budget of uncertainty in one analysis (in our case it is the scatter in the properties of the unknown inter-cluster WHIM), the image sum is a much faster approach than the source injection through the $u v w$-plane; however, it should be used with 


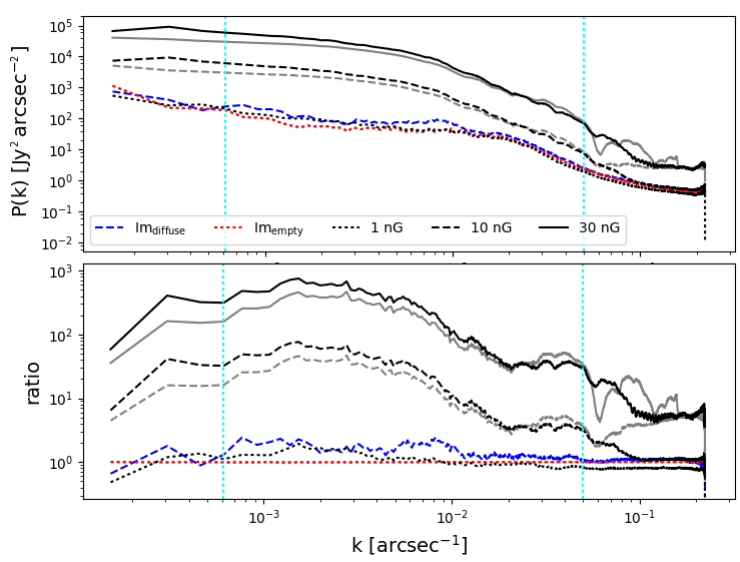

Fig. 9. Power spectrum of the injected filament shown in Fig. 4 for different $B_{0}$ models, as labelled. The vertical cyan dotted lines show the integration scale limits used to compute $P_{\mathrm{S}}$. They correspond respectively to about half the largest scale in the image $k^{-1}=2173^{\prime \prime} \sim$ 483 pixels and $k^{-1}=40^{\prime \prime}$ (corresponding to twice the synthesised beam full width at half maximum scale). The black lines show $P(k)$ resulting from the source injection into $u v w$ visibilities, whereas the grey lines show the result from the image-plane addition of the simulated image onto $\operatorname{Im}_{\text {empty }}$. Lower panel: same power spectra as in the upper panel, divided by the $\mathrm{Im}_{\mathrm{empty}}$ line.

caution as results are biased by a different sampling of the scales. The strength of the bias depends either on the sampling (window) function or the source power spectrum.

As a final caveat, our analysis assumes that for strong shocks in and around filaments, the acceleration efficiency of electrons is the one suggested by DSA (i.e. $\xi_{\mathrm{e}} \sim 10^{-2}$ ). This assumes, in turn, that despite the rather low particle density and magnetisation, shocks can form and undergo particle acceleration similar to what is already observed for the outer regions of galaxy clusters in the form of giant radio relics (see van Weeren et al. 2019, for a review). Moreover, our analysis assumes that the acceleration of electrons at shocks can proceed independently on the obliquity between the upstream magnetic field and the shock normal. However, recent numerical works by Banfi et al. (2020) show that shocks surrounding the cosmic web are more often quasi-perpendicular than random chance, as an effect to the peculiar gas velocity flow following the formation of filaments. In this case the vast majority of shocks in filaments are quasiperpendicular, and thus likely to be suitable for efficient electron acceleration (Xu et al. 2020). Furthermore, Masters et al. (2017) report a significant electron acceleration from the strong quasi-parallel shock while crossing the Saturn bow shock by the Cassini space mission (i.e. in plasma conditions similar to the intra-cluster medium). The acceleration seems to occur in the portion of the shock where upstream cosmic-ray streaming instabilities generate perpendicular small-scale magnetic field components, leading to particle acceleration.

\section{Conclusions}

In this work we attempted for the first time to combine dedicated LOFAR-HBA observations of inter-cluster filaments and numerical simulations of the magnetic cosmic web in order to derive upper limits on the magnetisation of the WHIM.

While our LOFAR observations do detect patches of diffuse emission of unclear origin, their morphology does not allow us to firmly associate the origin of the most prominent ones with the cosmic web. However, the presence of a faint diffuse large-

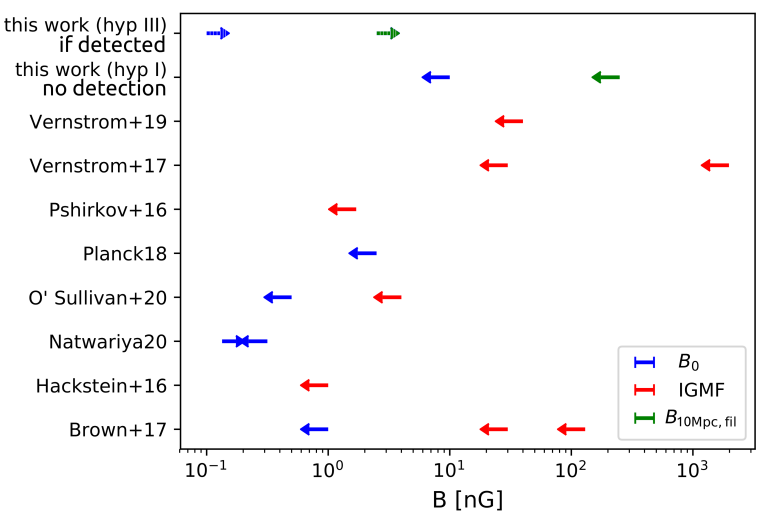

Fig. 10. Summary of the current upper and lower limits to the volumeaveraged IGMF (red arrows), $B$ in the filaments' WHIM (green arrows), and $B_{0}$ (blue arrows).

scale excess in comparison with numerical models allows us to derive inferences on the average magnetisation of such filaments, and possibly on the allowed initial amplitude of primordial seed magnetic fields. As the main outcome of our work, by fixing $\xi_{\mathrm{e}}=0.01$ for strong shocks, we derive an upper limit for the median magnetic field strength in filaments connecting massive galaxy clusters: $B_{10 \mathrm{Mpc}}<0.25 \mu \mathrm{G}$. Based on the dynamical evolution of magnetic fields given by present simulations (which is mostly dominated by simple compression of magnetic field lines), this also implies an upper limit of $B_{0}<10 \mathrm{nG}$ on the amplitude of primordial seed fields. The estimates above rely on the assumption that our observations constitute non-detections of diffuse emission from the cosmic web (hypothesis I).

As a mutually exclusive interpretation of our data, if some of the detected emission partially came from the shocked WHIM (hypothesis III), this would imply a median magnetic field of order of $B_{10 \mathrm{Mpc}} \geq \mathrm{nG}$ (see e.g. Fig. 6). This would be an important outcome as it might also indicate primordial magnetic fields with intensity $B_{0} \geq 0.1 \mathrm{nG}$.

The hypothesis that all of the excess diffuse emission detected in our maps came from the cosmic web (hypothesis II) can be easily rejected even by a visual check of some of the sources. Given the uncertainties connected to our method and the limited statistics of the detections in our sample, we favour the first interpretation of our results (hypothesis I, setting $B_{10 \mathrm{Mpc}}<0.25 \mu \mathrm{G}$ and $\left.B_{0}<10 \mathrm{nG}\right)$.

To compare our new limits with those of other recent works (Hackstein et al. 2016; Pshirkov et al. 2016; Vernstrom et al. 2017, 2019; Brown et al. 2017; O'Sullivan et al. 2020; Natwariya 2021, for a review, and Paoletti \& Finelli 2019 for joint BICEP2/Keck - Planck 2018 updated results), we show them in Fig. 10 separating the limits inferred for the IGMF or the magnetic field intensity of the WHIM (red arrows) and for the primordial magnetic field intensity $B_{0}$ (blue arrows). We note that our limits are still in agreement with the recent limits $0.134<B_{0} / \mathrm{nG}<0.316$ set by the level of excess diffuse emission observed by the ARCADE2 and EDGES $21 \mathrm{~cm}$ line experiments (Natwariya 2021). Furthermore, an apparent tension seems to arise between our lower limit to $B_{10 \mathrm{Mpc}}$ into filaments and the that derived from other probes such as the level of anisotropy in the arrival direction of charged ultra-high-energy cosmic rays used to limit the average amplitude of magnetic fields in voids to $\leq 1 \mathrm{nG}$ (Hackstein et al. 2016), or from the non-detection of a trend of rotation measures from distant radio sources with respect to redshifts (Pshirkov et al. 2016). Although they are 
computed over similar linear scales $(\geq \mathrm{Mpc})$ and globally refer to the IGM, they can still hardly be directly compared since referred to different IGM environments (e.g. voids, filaments, averaged). Interestingly, a recent work suggests that primordial magnetic fields with amplitude $\sim 0.1 \mathrm{nG}$ would possibly alleviate the existing tension between cosmological and standard candle-based estimates of $H_{0}$ (Jedamzik \& Pogosian 2020).

While it is hard to derive conclusive limits from these data, as no robust detection (although tentative) of the diffuse emission from the cosmic web can be claimed, this first attempt highlights the potential of low-frequency radio observations in constraining extragalactic magnetic fields, and its relevance to the study of cosmic magnetogenesis. With the analysis and the values obtained in this work, we can forecast that we will produce tighter constraints than those posed by CMB experiments by covering a $\sim \times 10$ larger sample of cluster pairs similar to the ones analysed here, even in the case of other non-detections.

Acknowledgements. We thank Klaus Dolag for providing corrections and helpful scientific feedback. NL and FV acknowledge financial support from the ERC Starting Grant "MAGCOW", no. 714196. ABon acknowledges financial support from the ERC Starting Grant "DRANOEL", no. 714245. ABot acknowledges support from the VIDI research programme with project number 639.042.729, which is financed by the Netherlands Organisation for Scientific Research (NWO). Radio imaging made use of WSClean v2.6 (Offringa et al. 2014) and DDF (Tasse et al. 2018). NL wishes to thank S. Carozzi and A. Crotti for psychological aid during and after the lock-down that followed the COVID-19 pandemic situation, when this work took shape. This paper is based (in part) on data obtained with the International LOFAR Telescope (obs. ID LC9_020, PI F.V.) and analysed using LOFAR-IT infrastructure. LOFAR (van Haarlem et al. 2013) is the Low Frequency Array designed, constructed by ASTRON and collectively operated by the ILT foundation. These data were (partly) processed by the LOFAR Two-Metre Sky Survey (LoTSS) team. This team made use of the LOFAR direction independent calibration pipeline (https://github.com/lofar-astron/prefactor) which was deployed by the LOFAR e-infragroup on the Dutch National Grid infrastructure with support of the SURF Co-operative through grants e-infra 160022 and e-infra 160152 (Mechev et al. 2017, PoS(ISGC2017)002). The cosmological simulations were performed with the ENZO code (http://enzo-project.org), which is the product of a collaborative effort of scientists at many universities and national laboratories. FV acknowledges the usage of computational resources on the Piz Daint supercomputer at CSCS-ETHZ (Lugano, Switzerland) under project s805, and he also acknowledges the usage of online storage tools kindly provided by the Inaf Astronomica Archive (IA2) initiave (http://www .ia2 .inaf.it).

\section{References}

Aghanim, N., Douspis, M., Hurier, G., et al. 2019, A\&A, 632, A47 Akahori, T., Gaensler, B. M., \& Ryu, D. 2014, ApJ, 790, 123

Allington-Smith, J. R., Ellis, R., Zirbel, E. L., \& Oemler, A., Jr. 1993, ApJ, 404, 521

Banfi, S., Vazza, F., \& Wittor, D. 2020, MNRAS, 496, 3648

Banfi, S., Vazza, F., \& Gheller, C. 2021, MNRAS, 503, 4016

Becker, R. H., White, R. L., \& Helfand, D. J. 1995, ApJ, 450, 559

Beresnyak, A., \& Miniati, F. 2016, ApJ, 817, 127

Biff, V., Planelles, S., Borgani, S., et al. 2018, MNRAS, 476, 2689

Botteon, A., van Weeren, R. J., Brunetti, G., et al. 2020a, MNRAS, 499, L11

Botteon, A., Brunetti, G., Ryu, D., \& Roh, S. 2020b, A\&A, 634, A64

Briggs, D. S. 1995, Am. Astron. Soc. Meet. Abstr., 187, 112.02

Brown, S., Vernstrom, T., Carretti, E., et al. 2017, MNRAS, 468, 4246

Brüggen, M., Ruszkowski, M., Simionescu, A., Hoeft, M., \& Dalla Vecchia, C. 2005, ApJ, 631, L21

Bryan, G. L., Norman, M. L., O’Shea, B. W., et al. 2014, ApJS, 211, 19

Bykov, A. M., Vazza, F., Kropotina, J. A., Levenfish, K. P., \& Paerels, F. B. S. 2019a, Space Sci. Rev., 215, 14
Bykov, A. M., Petrov, A. E., Krassilchtchikov, A. M., et al. 2019b, ApJ, 876, L8 Cen, R., \& Ostriker, J. P. 1999, ApJ, 514, 1

Colberg, J. M., Krughoff, K. S., \& Connolly, A. J. 2005, MNRAS, 359, 272

Davé, R., Cen, R., Ostriker, J. P., et al. 2001, ApJ, 552, 473

Dedner, A., Kemm, F., Kröner, D., et al. 2002, J. Comput. Phys., 175, 645

de Gasperin, F., Dijkema, T. J., Drabent, A., et al. 2019, A\&A, 622, A5

Dolag, K., Bartelmann, M., \& Lesch, H. 1999, A\&A, 348, 351

Donnert, J., Vazza, F., Brüggen, M., \& ZuHone, J. 2018, Space Sci. Rev., 214, 122

Gheller, C., \& Vazza, F. 2019, MNRAS, 486, 981

Gheller, C., Vazza, F., Brüggen, M., et al. 2016, MNRAS, 462, 448

Gheller, C., Vazza, F., \& Bonafede, A. 2018, MNRAS, 480, 3749

Govoni, F., Orrù, E., Bonafede, A., et al. 2019, Science, 364, 981

Hackstein, S., Vazza, F., Brüggen, M., Sigl, G., \& Dundovic, A. 2016, MNRAS, 462, 3660

Hoeft, M., \& Brüggen, M. 2007, MNRAS, 375, 77

Jedamzik, K., \& Pogosian, L. 2020, Phys. Rev. Lett., 125, 181302

Kang, H., Ryu, D., \& Jones, T. W. 2012, ApJ, 756, 97

Locatelli, N., Vazza, F., \& Domínguez-Fernández, P. 2018, Galaxies, 6, 128

Macquart, J. P., Prochaska, J. X., McQuinn, M., et al. 2020, Nature, 581, 391

Masters, A., Sulaiman, A. H., Stawarz, Ł., et al. 2017, ApJ, 843, 147

Mechev, A., Oonk, J. B. R., Danezi, A., et al. 2017, Proceedings of the International Symposium on Grids and Clouds (ISGC) 2017, 2

Mohan, N., \& Rafferty, D. 2015, Astrophysics Source Code Library [record ascl:1502.007]

Natwariya, P. K. 2021, Eur. Phys. J. C, 81, 394

Nicastro, F., Kaastra, J., Krongold, Y., et al. 2018, Nature, 558, 406

Offringa, A. R., McKinley, B., Hurley-Walker, N., et al. 2014, MNRAS, 444, 606

O'Sullivan, S. P., Brüggen, M., Vazza, F., et al. 2020, MNRAS, 495, 2607

Paoletti, D., \& Finelli, F. 2019, JCAP, 2019, 028

Piffaretti, R., Arnaud, M., Pratt, G. W., Pointecouteau, E., \& Melin, J. B. 2011, A\&A, 534, A109

Prestage, R. M., \& Peacock, J. A. 1988, MNRAS, 230, 131

Pshirkov, M. S., Tinyakov, P. G., \& Urban, F. R. 2016, Phys. Rev. Lett., 116, 191302

Reiprich, T. H., Veronica, A., Pacaud, F., et al. 2021, A\&A, 647, A2

Ryu, D., Kang, H., Cho, J., \& Das, S. 2008, Science, 320, 909

Shimwell, T. W., Röttgering, H. J. A., Best, P. N., et al. 2017, A\&A, 598, A104

Shimwell, T. W., Tasse, C., Hardcastle, M. J., et al. 2019, A\&A, 622, A1

Smirnov, O. M., \& Tasse, C. 2015, MNRAS, 449, 2668

Stuardi, C., Bonafede, A., Wittor, D., et al. 2019, MNRAS, 489, 3905

Stuardi, C., O'Sullivan, S. P., Bonafede, A., et al. 2020, A\&A, 638, A48

Tanimura, H., Aghanim, N., Kolodzig, A., Douspis, M., \& Malavasi, N. 2020, A\&A, 643, L2

Tasse, C. 2014a, ArXiv e-prints [arXiv:1410.8706]

Tasse, C. 2014b, A\&A, 566, A127

Tasse, C., Hugo, B., Mirmont, M., et al. 2018, A\&A, 611, A87

Tasse, C., Shimwell, T., Hardcastle, M. J., et al. 2021, A\&A, 648, A1

Uchiyama, Y., Aharonian, F. A., Tanaka, T., Takahashi, T., \& Maeda, Y. 2007. Nature, 449, 576

Vacca, V., Oppermann, N., Enßlin, T., et al. 2016, A\&A, 591, A13

van Haarlem, M. P., Wise, M. W., Gunst, A. W., et al. 2013, A\&A, 556, A2

van Weeren, R. J., Williams, W. L., Hardcastle, M. J., et al. 2016, ApJS, 223, 2

van Weeren, R. J., de Gasperin, F., Akamatsu, H., et al. 2019, Space Sci. Rev., 215,16

Vazza, F., Brüggen, M., Gheller, C., \& Wang, P. 2014, MNRAS, 445, 3706

Vazza, F., Ferrari, C., Brüggen, M., et al. 2015, A\&A, 580, A119

Vazza, F., Brüggen, M., Gheller, C., et al. 2017, Classical Quantum Gravity, 34, 234001

Vazza, F., Ettori, S., Roncarelli, M., et al. 2019, A\&A, 627, A5

Vernstrom, T., Gaensler, B. M., Brown, S., Lenc, E., \& Norris, R. P. 2017, MNRAS, 467, 4914

Vernstrom, T., Gaensler, B. M., Rudnick, L., \& Andernach, H. 2019, ApJ, 878, 92

Vernstrom, T., Heald, G., Vazza, F., et al. 2021, MNRAS, 505, 4178

Williams, W. L., van Weeren, R. J., Röttgering, H. J. A., et al. 2016, MNRAS, 460, 2385

Wing, J. D., \& Blanton, E. L. 2011, AJ, 141, 88

Xu, R., Spitkovsky, A., \& Caprioli, D. 2020, ApJ, 897, L41

Zirbel, E. L. 1997, ApJ, 476, 489 